\title{
Automated Verification of Quantum Protocols using MCMAS
}

\author{
F. Belardinelli, P. Gonzalez, A. Lomuscio \\ \{f.belardinelli, pavel.gonzalez09, a.lomuscio\}@imperial.ac.uk \\ Imperial College London \\ London, UK
}

\begin{abstract}
We present a methodology for the automated verification of quantum protocols using MCMAS, a symbolic model checker for multi-agent systems [17]. The method is based on the logical framework developed by D'Hondt and Panangaden [10] for investigating epistemic and temporal properties, built on the model for Distributed Measurement-based Quantum Computation (DMC) [9], an extension of the Measurement Calculus [8] to distributed quantum systems. We describe the translation map from DMC to interpreted systems, the typical formalism for reasoning about time and knowledge in multi-agent systems [14]. Then, we introduce DMC2ISPL, a compiler into the input language of the MCMAS model checker [17]. We demonstrate the technique by verifying the Quantum Teleportation Protocol, and discuss the performance of the tool.
\end{abstract}

\section{Introduction}

Quantum computing has gained prominence in the last decade due to theoretical advances as well as applications to security, information processing, and simulation of quantum mechanical systems [19]. With this increase of activity, the need for validation of correctness of quantum algorithms has arisen. Model checking has shown to be a promising verification technique [6]. However, tools and techniques for model checking both temporal and epistemic properties of quantum systems have not been developed yet. In this paper we aim to bridge this gap by introducing a methodology for the automated verification of quantum protocols using MCMAS [17], a symbolic model checker for multi-agent systems (MAS).

The fundamental question from an epistemic point of view is how to model a flow of quantum information. Is it meaningful to talk about "quantum knowledge"? And if it is, how can we express this concept? Several logics, which can be used for reasoning about knowledge in the context of distributed quantum computation, have been recently suggested. One of the first attempts was based on Quantum Message Passing Environments [18]. A different approach, i.e. Quantum Dynamic-Epistemic Logic [1, 2, 3], was developed to model the behaviour of quantum systems. A third account [7, 10, 11] was built on the Distributed Measurement-based Quantum Computation [9], which extends the Measurement Calculus [8], a formal model for one-way quantum computations. Among these accounts, the logic based on Distributed Measurement-based Quantum Computation (DMC) has an underlying operational semantics similar to the semantics of interpreted systems [14]. This makes it suitable for model checking using MCMAS. However, interpreted systems (IS) have a Boolean semantics, which requires us to abstract from the underlying probability distribution. While we recognise that a full analysis of quantum phenomena requires stochastic considerations, we believe there are still useful lessons to be learned about protocols when these are abstracted from. The point of the paper is partly to explore this hypotheses.

In this paper we describe a translation from DMC to IS. We also report on a source-to-source compiler that performs the translation into the input language of MCMAS. The compiler enables the use of MCMAS to verify automatically temporal and epistemic properties of quantum protocols specified in DMC. We verify the Quantum Teleportation protocol [5] against the properties stated and informally proved in [10], and show that one specification does not hold contrary to the paper's claim.

M. Massink and H. Wiklicky (Eds.):Workshop on Quantitative Aspects of Programming Languages and Systems 2012 (QAPL 2012)

EPTCS 85, 2012, pp. 48-62 doi 10.4204/EPTCS.85.4 (c) Belardinelli, Gonzalez, Lomuscio

This work is licensed under the Creative Commons Attribution License. 
Related Work. Several approaches to model checking quantum systems have already appeared in the literature. To our knowledge, the only dedicated verification tool for quantum protocols is the Quantum Model Checker (QMC) [15]. The model checker supports specifications in quantum computational temporal logic (QCTL), but quantum operators are restricted to the Clifford group, which is the normalizer of the group of Pauli operators [19]. Although it contains many common operators, quantum circuits that involve only Clifford group operators are not universal. Such circuits can be simulated in polynomial time on a classical computer; however, this leads to a loss of expressive power.

In the same research line [20] a theoretical framework to model check LTL properties using quantum automata is proposed, and an algorithm for checking invariants of quantum systems is presented. Finally, in [13] the Quantum Key Distribution (QKD) protocol is verified against specific eavesdropping security properties. The authors elaborate an ad hoc model of the protocol, that they analyse using PRISM [16].

However, we stress that none of these contributions explicitly deal with knowledge. So, these approaches do not allow the verification of the temporal epistemic properties discussed in [10].

Structure. Organizationally, Section 2 gives an overview of the Distributed Measurement-based Quantum Computation, Interpreted Systems, and Quantum Epistemic Logic. Section 3 presents a methodology for translating a protocol specified in DMC into the corresponding IS. Section 4 describes and evaluates an implementation of the formal methodology. Section 5 offers brief conclusions.

\section{Preliminaries}

We discuss only the issues directly related to the paper and refer the reader to the relevant references for an in-depth coverage of these topics. We assume familiarity with the concepts of quantum computation [19].

\subsection{Distributed Measurement-based Quantum Computation}

At the heart of the Measurement Calculus are measurement patterns [8]. A pattern $\mathscr{P}=(V, I, O, \mathscr{A})$ consists of a computation space $V$, which contains all qubits involved in the execution of $\mathscr{P}$, a set $I$ of input qubits, a set $O$ of output qubits, and a finite sequence $\mathscr{A}$ of commands $A_{p} \ldots A_{1}$, which are applied to qubits in $V$ from right to left. The possible commands are the entanglement operator $E_{q r}$, the measurement $M_{q}^{\alpha}$, and the corrections $X_{q}$ and $Z_{q}$, where $q$ and $r$ represent the qubits on which these commands operate, and $\alpha$ is a measurement angle in $[0,2 \pi]$.

An agent $\mathbf{A}$ [9], denoted as $\mathbf{A}(\mathbf{i}, \mathbf{o}): Q . \mathscr{E}$, is characterised by its classical input $\mathbf{i}$ and output $\mathbf{o}$, by a set $Q$ of qubits, and by a finite event sequence $\mathscr{E}$, which consists of patterns and commands for classical (c? $x, \mathrm{c} ! y)$ and quantum (qc?x, qc!q) communication. A network $\mathscr{N}$ of agents [9] is defined as a set of concurrently acting agents, together with the global quantum state $\sigma$, specifically $\mathscr{N}=\mathbf{A}_{1}\left(\mathbf{i}_{1}, \mathbf{o}_{1}\right)$ : $Q_{1} \mathscr{E}_{1}|\ldots| \mathbf{A}_{m}\left(\mathbf{i}_{m}, \mathbf{o}_{m}\right): Q_{m} . \mathscr{E}_{m} \| \sigma$, abbreviated as $\mathscr{N}={ }_{i} \mathbf{A}_{i}\left(\mathbf{i}_{i}, \mathbf{o}_{i}\right): Q_{i} \cdot \mathscr{E}_{i} \| \sigma$. The configuration $C$ of a network $\mathscr{N}$ at a particular point in time is described by a set of agents, their classical local states, and the quantum state $\sigma$, formally $C=\sigma, \Gamma_{1}, \mathbf{a}_{1}\left|\Gamma_{2}, \mathbf{a}_{2}\right| \ldots \mid \Gamma_{m}, \mathbf{a}_{m}$, abbreviated as $C=\sigma,\left.\right|_{i} \Gamma_{i}, \mathbf{a}_{i}$, where $\Gamma_{i}$ represents the classical state of agent $\mathbf{a}_{i}$, which is defined as a partial mapping from classical variables to values. The set $\mathscr{C}_{\mathscr{N}}$ contains all configurations that potentially occur during the execution of the network $\mathscr{N}$.

Operational and denotational semantics for DMC are defined in [9]; however, here we are more interested in its small-step semantics. The following small-step rules for configuration transitions describe how the network evolves over time. If the quantum state does not change in an evaluation step, the writing $\sigma \vdash$ precedes the rule. Also, we use a shorthand notation for agents: $\mathbf{a}_{i}=\mathbf{A}_{i}: Q_{i} . \mathscr{E}_{i}, \mathbf{a}_{i} . E=\mathbf{A}_{i}: Q_{i} \cdot\left[\mathscr{E}_{i} . E\right]$, 
$\mathbf{a}^{-q}=\mathbf{A}: Q \backslash q \cdot \mathscr{E}$, and $\mathbf{a}^{+q}=\mathbf{A}: Q \uplus q \cdot \mathscr{E}[q / x]$, where $E$ is some event.

$$
\begin{gathered}
\frac{\sigma, \mathscr{P}(V, I, O, \mathscr{A}) \longrightarrow_{\lambda} \sigma^{\prime}, \Gamma^{\prime}}{\sigma, \Gamma, \mathbf{A}: I \uplus R \cdot[\mathscr{E} \cdot \mathscr{P}] \Longrightarrow_{\lambda} \sigma^{\prime}, \Gamma \cup \Gamma^{\prime}, \mathbf{A}: O \uplus R \cdot \mathscr{E}} \\
\frac{\Gamma_{2}(y)=v}{\sigma \vdash\left(\Gamma_{1}, \mathbf{a}_{1} \cdot \mathrm{c} ? x\left|\Gamma_{2}, \mathbf{a}_{2} \cdot \mathrm{c} ! y \Longrightarrow \Gamma_{1}[x \mapsto v], \mathbf{a}_{1}\right| \Gamma_{2}, \mathbf{a}_{2}\right)} \\
\frac{L \vdash\left(\Gamma_{1}, \mathbf{a}_{1} \cdot \mathrm{qc} ? x\left|\Gamma_{2}, \mathbf{a}_{2} \cdot \mathrm{qc} ! q \Longrightarrow \Gamma_{1}, \mathbf{a}_{1}^{+q}\right| \Gamma_{2}, \mathbf{a}_{2}^{-q}\right)}{C_{\lambda} R} \\
\frac{L\left|L^{\prime} \Longrightarrow_{\lambda} R\right| L^{\prime}}{}
\end{gathered}
$$

The first rule refers to local operations. Since a pattern's big-step semantics is given by a probabilistic transition system, described by $\longrightarrow$, a probability $\lambda$ is introduced here. Also, an agent changes its sort depending on the pattern's output $O$. The next two rules are for the classical and the quantum rendezvous. For the quantum rendez-vous a substitution $q$ for $x$ in the event sequence of the receiving agent is performed and agents need to update their qubit sorts. (4) is a metarule, which is required to express that any of the other rules may fire in the context of a larger system.

\subsection{Interpreted Systems and MCMAS}

Interpreted systems [14] are the typical formalism for reasoning about time and knowledge in multi-agent systems. In IS each agent $i$ from a non-empty set $A g$ of agents is modelled by a set of local states $L_{i}$, a set of actions $A c t_{i}$ that she may perform according to her protocol function $P_{i}$, and an evolution function $t_{i}$. A special agent $E$, representing the environment in which the other agents operate, is also described by a set of local states $L_{E}$, a set of actions $A c t_{E}$, a protocol $P_{E}$, and an evolution function $t_{E}$. For every $j \in A g \cup\{E\}$, the protocol $P_{j}$ is defined as a function $P_{j}: L_{j} \rightarrow 2^{A c t_{j}}$, assigning a set of actions to aiven local state. Intuitively, $\alpha_{j} \in P_{j}\left(l_{j}\right)$ means that action $\alpha_{j}$ is enabled in $l_{j}$. The evolution function $t_{j}$ is a transition function returning the target local state given the current local state and the set of actions performed by all agents, formally $t_{j}: L_{j} \times A c t_{1} \times \cdots \times A c t_{n} \times A c t_{E} \rightarrow L_{j}$ under the constraint $\alpha_{j} \in P_{j}\left(l_{j}\right)$. Agents evolve simultaneously in every state of the system according to the joint transition function $t$.

The set Act of joint actions is defined as the Cartesian product of all agents' actions, formally $A c t=A c t_{1} \times \cdots \times A c t_{n} \times A c t_{E}$. The Cartesian product $S=L_{i} \times \cdots \times L_{n} \times L_{E}$ of the agents' local states is the set of all global states of the system. The local state of agent $i$ in the global state $g \in S$ is denoted as $l_{i}(g)$. The description of an interpreted system is concluded by including a set of atomic propositions $A P=\left\{p_{1}, p_{2}, \ldots\right\}$ and an evaluation relation $V \subseteq A P \times S$. Formally, an interpreted system is defined as a tuple $I S=\left\langle\left(L_{i}, A c t_{i}, P_{i}, t_{i}\right)_{i \in A g},\left(L_{E}, A c t_{E}, P_{E}, t_{E}\right), V\right\rangle$.

Interpreted systems can be used to interpret CTLK, a logic combining the branching-time temporal logic CTL with epistemic modalities. The formal language $\mathscr{L}$ is built from propositional atoms $p \in A P$ and agents $i \in A g$ as follows:

$$
\varphi::=p|\neg \varphi| \varphi \vee \varphi|E X \varphi| E G \varphi|E \varphi U \psi| K_{i}
$$

The formulae in $\mathscr{L}$ have the following intuitive meaning. $E X \varphi$ : there is a path where $\varphi$ holds in the next state; $E G \varphi$ : there is a path where $\varphi$ always holds; $E \varphi U \psi$ : there is a path where $\varphi$ holds at least until at some state $\psi$ holds; $K_{i} \varphi$ : agent $i$ knows $\varphi$. The other standard CTL formulae, e.g., $A F \varphi$ : for all paths $\varphi$ eventually holds, can be derived from the above. The formal definition of satisfaction in interpreted systems follows. 
In an interpreted system $\mathscr{M}$ the evolution function $t$ determines a transition relation $\stackrel{\mathscr{M}}{\longrightarrow}$ on states such that $s \stackrel{\mathscr{M}}{\longrightarrow} s^{\prime}$ iff there is a joint action $\alpha \in$ Act such that $t(s, \alpha)=s^{\prime}$. A path $\pi$ is an infinite sequence of states $s_{0} \stackrel{\mathscr{M}}{\longrightarrow} s_{1} \stackrel{\mathscr{M}}{\longrightarrow} \ldots$. Further, $\pi^{n}$ denotes the $n$-th state in the sequence, i.e, $s_{n}$. Finally, for each agent $i \in A g$, we introduce the epistemic equivalence relation $\sim_{i}^{\mathscr{M}}$ such that $s \sim_{i}^{\mathscr{M}} s^{\prime}$ iff $l_{i}(s)=l_{i}\left(s^{\prime}\right)$.

Given the IS $\mathscr{M}$, a state $s$, and a formula $\phi \in \mathscr{L}$, the satisfaction relation $\vDash$ is defined as follows:
$(\mathscr{M}, s) \vDash p \quad$ iff $V(p, s)$
$(\mathscr{M}, s) \vDash \neg \phi \quad$ iff $\quad(\mathscr{M}, s) \not \models \phi$
$(\mathscr{M}, s) \vDash \phi \vee \phi^{\prime} \quad$ iff $\quad(\mathscr{M}, s) \vDash \phi$ or $(\mathscr{M}, s) \vDash \phi^{\prime}$
$(\mathscr{M}, s) \vDash E X \phi \quad$ iff there is a path $\pi$ such that $\pi^{0}=s$, and $\left(\mathscr{M}, \pi^{1}\right) \vDash \phi$
$(\mathscr{M}, s) \vDash E G \phi \quad$ iff there is a path $\pi$ such that $\pi^{0}=s$, and for all $n \in \mathbb{N},\left(\mathscr{M}, \pi^{n}\right) \vDash \phi$
$(\mathscr{M}, s) \vDash E \phi U \phi^{\prime}$ iff there is a path $\pi$ such that $\pi^{0}=s$, for some $n \in \mathbb{N},\left(\mathscr{M}, \pi^{n}\right) \vDash \phi^{\prime}$, and for all $n^{\prime}, 0 \leq n^{\prime}<n$ implies $\left(\mathscr{M}, \pi^{n^{\prime}}\right) \vDash \phi$
$(\mathscr{M}, s) \vDash K_{i} \phi \quad$ iff for all $s^{\prime} \in S, s \sim_{i}^{\mathscr{M}} s^{\prime}$ implies $\left(\mathscr{M}, s^{\prime}\right) \vDash \phi$

A formula $\phi \in \mathscr{L}$ is true in an IS $\mathscr{M}$, or $\mathscr{M} \vDash \phi$, iff for all $s \in S,(\mathscr{M}, s) \vDash \phi$.

In [17] the authors present a methodology for the verification of IS based on model checking [6] via ordered binary decision diagrams. These verification techniques have been implemented in the MCMAS model checker. The input to the model checker is given as an Interpreted Systems Programming Language (ISPL) program, which is essentially a machine readable IS.

\subsection{Quantum Epistemic Logic}

A formal framework for reasoning about temporal and epistemic properties of distributed quantum systems was developed in [10] on top of DMC. The authors argue that quantum knowledge is not a meaningful concept, but it is of interest to reason about classical knowledge pertaining to a quantum system. In this sense, the quantum information possessed by an agent concerns the qubits she owns, the local operations she applies to these qubits, the non-local entanglement she shares initially, and possibly prior knowledge of her local quantum inputs. All this information is contained in her local state $\Gamma_{i}$ and her event sequence $\mathscr{E}_{i}$. Given a network $\mathscr{N}$, the epistemic accessibility relation $\sim_{i}^{\mathscr{N}}$ for an agent $\mathbf{A}_{i}$ is defined in [10] as follows: for all configurations $C=\sigma,\left.\right|_{i} \Gamma_{i}, \mathbf{A}_{i}: Q_{i} \cdot \mathscr{E}_{i}$ and $C^{\prime}=\sigma^{\prime},\left.\right|_{i} \Gamma_{i}^{\prime}, \mathbf{A}_{i}: Q_{i}^{\prime} \cdot \mathscr{E}_{i}^{\prime}$ in $\mathscr{C}_{\mathscr{N}}, C$ and $C^{\prime}$ are indistinguishable to agent $\mathbf{A}_{i}$, written as $C \sim_{i}^{\mathscr{N}} C^{\prime}$, if $\Gamma_{i}=\Gamma_{i}^{\prime}$ and $\mathscr{E}_{i}=\mathscr{E}_{i}^{\prime}$. The semantics for the modal operator $K_{i}$ for the knowledge of agent $\mathbf{A}_{i}$ is then defined in the usual way: $(C, \mathscr{N}) \vDash K_{i} \varphi$ iff for all $C^{\prime}$, $C^{\prime} \sim_{i}^{\mathscr{N}} C$ implies $\left(C^{\prime}, \mathscr{N}\right) \vDash \varphi$.

We now give the truth conditions for all formulae in $\mathscr{L}$ in a network $\mathscr{N}$. The set of atomic propositions $A P=\left\{x=v, x=y, \mathbf{A}_{i}\right.$ has $\left.q, q_{1} \ldots q_{n}=|\psi\rangle, q_{i}=q_{j}\right\}$ is considered in [7]. In a configuration $C$ of a network $\mathscr{N}$ the truth conditions for these atomic propositions are given as follows:
$(C, \mathscr{N}) \vDash x=v$
iff there is an agent $i$ such that $\Gamma_{i}(x)=v$
$(C, \mathscr{N}) \vDash x=y$
iff there are agents $i, j$ such that $\Gamma_{i}(x)=\Gamma_{i}(y)$
$(C, \mathscr{N}) \vDash \mathbf{A}_{i}$ has $q \quad$ iff $q \in Q_{i}$
$(C, \mathscr{N}) \vDash q_{1} \ldots q_{n}=|\psi\rangle$ iff $q_{1} \ldots q_{n}=|\psi\rangle$
$(C, \mathscr{N}) \vDash q_{i}=q_{j} \quad$ iff there is $|\psi\rangle$ such that $|\psi\rangle=q_{i}=q_{j}$

In networks the small-step rules given in Section 2.1 determine a transition relation $\stackrel{\mathscr{N}}{\longrightarrow}$ such that $C \stackrel{\mathscr{N}}{\longrightarrow} C^{\prime}$ iff there is a rule that applied to $C$ returns $C^{\prime}$. A path $\gamma$ is an infinite sequence of configurations $C_{0} \stackrel{\mathscr{N}}{\longrightarrow} C_{1} \stackrel{\mathscr{N}}{\longrightarrow} \ldots$. Further, $\gamma^{n}$ denotes the $n$-th state in the sequence, i.e, $C_{n}$. Finally, for each agent $\mathbf{A}_{i}$ 
in the network, we introduce the epistemic equivalence relation $\sim_{i}^{\mathcal{N}}$ such that $C \sim_{i}^{\mathcal{N}} C^{\prime}$ iff $\Gamma_{i}=\Gamma_{i}^{\prime}$ and $\mathscr{E}_{i}=\mathscr{E}_{i}^{\prime}$

Given the network $\mathscr{N}$, a configuration $C$, and a formula $\phi \in \mathscr{L}$, the satisfaction relation $\vDash$ is defined as follows:

$$
\begin{array}{lll}
(C, \mathscr{N}) \vDash p & \text { iff } \quad C \text { satisfies the corresponding condition above for atomic } p \in A P \\
(C, \mathscr{N}) \vDash \neg \phi \quad \text { iff } \quad(C, \mathscr{N}) \not \phi & \\
(C, \mathscr{N}) \vDash \phi \vee \phi^{\prime} & \text { iff } \quad(C, \mathscr{N}) \vDash \phi \text { or }(C, \mathscr{N}) \vDash \phi^{\prime} \\
(C, \mathscr{N}) \vDash E X \phi \quad \text { iff } & \text { there is a path } \gamma \text { such that } \gamma^{0}=C, \text { and }\left(\gamma^{1}, \mathscr{N}\right) \vDash \phi \\
(C, \mathscr{N}) \vDash E G \phi \quad \text { iff } & \text { there is a path } \gamma \text { such that } \gamma^{0}=C, \text { and for all } n \in \mathbb{N},\left(\gamma^{n}, \mathscr{N}\right) \vDash \phi \\
(C, \mathscr{N}) \vDash E \phi U \phi^{\prime} & \text { iff } \quad \text { there is a path } \gamma \text { such that } \gamma^{0}=C, \text { for some } n \in \mathbb{N},\left(\gamma^{n}, \mathscr{N}\right) \vDash \phi^{\prime}, \\
& \quad \text { and for all } n^{\prime}, 0 \leq n^{\prime}<n \text { implies }\left(\gamma^{n^{\prime}}, \mathscr{N}\right) \vDash \phi \\
(C, \mathscr{N}) \vDash K_{i} \phi \quad \text { iff } \quad \text { for all } C^{\prime} \in \mathscr{N}, C \sim_{i}^{\mathscr{N}} C^{\prime} \text { implies }\left(C^{\prime}, \mathscr{N}\right) \vDash \phi
\end{array}
$$

A formula $\phi \in \mathscr{L}$ is true in a network $\mathscr{N}$, or $\mathscr{N} \vDash \phi$, iff for all configurations $C,(C, \mathscr{N}) \vDash \phi$.

\subsection{Quantum Teleportation Protocol}

The goal of the Quantum Teleportation Protocol (QTP) is to transmit a qubit from one party to another with the aid of an entangled pair of qubits and classical resources. For reasons of space we refer to [5] for a detailed presentation of QTP. The DMC specification of the protocol is given in [9] as:

$$
\mathscr{N}_{Q T P}=\mathbf{A}:\{1,2\} \cdot\left[\left(\mathrm{c} ! s_{2} s_{1}\right) \cdot M_{12}^{0,0} E_{12}\right] \mid \mathbf{B}:\{3\} \cdot\left[X_{3}^{x_{2}} Z_{3}^{x_{1}} \cdot\left(\mathrm{c} ? x_{2} x_{1}\right)\right] \| E_{23} .
$$

The informal reading is as follows: Alice $\mathbf{A}$ and $\mathrm{Bob} \mathbf{B}$ share the entangled pair $E_{23}$ of qubits 2 and 3, and Alice wants to transmit the input qubit 1 . In the first step, she entangles $\left(E_{12}\right)$ her qubits 1 and 2 . Then she measures $\left(M_{12}^{0,0}\right)$ both of them. Next, she sends via classical communication $\left(\mathrm{c} ! s_{2} s_{1}\right)$ the measurement outcomes to Bob. Upon receipt ( $c$ ? $\left.x_{2} x_{1}\right)$, Bob applies corrections $\left(X_{3}^{x_{2}} Z_{3}^{x_{1}}\right)$ to his qubit 3 depending on these measurements. The result is that Bob's qubit 3 is guaranteed to be in the same state as Alice's input qubit 1.

\section{Formal Mapping}

In this section we present a methodology for translating a protocol specified in DMC into the corresponding IS. Formally, we define a mapping $f: D M C \rightarrow I S$, such that $f$ preserves satisfaction of formulae in the specification language $\mathscr{L}$. First, we describe the translation of the global quantum state and classical states of agents. Then we cover the rules in DMC. Finally, we show that $f$ is sound.

\subsection{Classical States of Agents and Global Quantum State}

Given a network $\mathscr{N}$ we introduce an agent $i \in A g$ for each agent $\mathbf{A}_{i}\left(\mathbf{i}_{i}, \mathbf{o}_{i}\right): Q_{i} \cdot \mathscr{E}_{i}$ in $\mathscr{N}$, as well as the Environment agent $E$. We take a local state $l_{i} \in L_{i}$ of agent $i$ to be a tuple of vector variables $(\vec{x}, \vec{y}, \vec{s}, \vec{q}, p c)$ defined as follows:

- Each classical input bit in $\mathbf{i}_{i}$ is mapped to a variable $y \in l_{i}$ in the domain $\{0,1\}$.

- A bit received from an agent via the classical receive event c? $x$ in the event sequence $\mathscr{E}_{i}$ is mapped to a variable $x \in l_{i}$ in the domain $\{0,1, \perp\}$, where $\perp$ denotes the undefined value before communication. 
- A variable $s \in l_{i}$, called signal, represents the outcome of a measurement event $M_{q}^{\alpha}$ in the event sequence $\mathscr{E}_{i}$, where $q$ is the measured qubit and $\alpha$ is a measurement angle. A signal can attain values $\{0,1, \perp\}$, where $\perp$ denotes the undefined value of the signal before the agent executes the measurement.

- A variable $q \in l_{i}$ in the domain $\{0,1,2\}$ represents the ownership relation between agent $\mathbf{A}_{i}$ and qubit $q$ with the following meaning: if $\mathbf{A}_{i}$ is not in possession of $q$, i.e., $q \notin Q_{i}$, then we take $q=0$. If $\mathbf{A}_{i}$ owns the qubit $q$, i.e., $q \in Q_{i}$, then $q=1$ or $q=2$. The former value represents that $\mathbf{A}_{i}$ does not know the exact state of the qubit, the latter value represents that she knows it. We assume that the agent knows the state of the qubit once she measures it or prepares it in a specific state. This is motivated as there is classical information involved in both cases. However, the agent loses this knowledge when she sends the qubit to another agent, as it is no longer in her possession, or entangles it with another qubit. Note that correction commands preserve knowledge because they are deterministic actions that neither entangle nor separate qubits.

- $p c \in l_{i}$ is a counter for the events in the event sequence $\mathscr{E}_{i}$ executed by agent $\mathbf{A}_{i}$.

Example 1. Consider the specification of QTP in DMC as given in Section 2.4. The local state of Alice is described by the tuple $l_{A}=\left(s_{1}, s_{2}, q_{1}, q_{2}, q_{3}, p c\right)$, and similarly the local state of Bob is $l_{B}=\left(x_{1}, x_{2}, q_{1}, q_{2}, q_{3}, p c\right)$. In the initial state Alice owns the input qubits $q_{1}$ and $q_{2}$ in the entangled pair, while Bob owns the qubit $q_{3}$, and neither of them knows anything about their qubits. Alice has not yet measured any qubit nor has she sent anything to Bob. The program counters of both agents point to the first event in their event sequences. All this is captured in variable assignments $(\perp, \perp, 1,1,0,1)$ for Alice and $(\perp, \perp, 0,0,1,1)$ for Bob.

A local state $l_{E} \in L_{E}$ of the Environment represents the quantum state $\sigma$ of the network. $l_{E}$ is a tuple of vector variables $\left(\vec{q}, \vec{q}^{\prime}, \vec{e}, g c\right)$ defined as follows:

- We divide the global quantum state at any given time into the smallest possible substates - individual qubits and/or systems of entangled qubits - such that these are in pure states, i.e., they can be represented as a vector in a Hilbert space. We generate the reachable quantum state space of the network using the small-step rules for patterns and enumerate all such encountered substates. Thus, every reachable substate has an associated name $q s_{n}, n \in \mathbb{N}$.

- For every qubit $q \in \mathscr{N}$ we introduce a variable $q \in l_{E}$. The domain of $q$ is the set of names of quantum states that $q$ may attain in any run of the protocol, together with the value $\perp$ indicating that the qubit is not in a pure state but entangled with other qubits.

- Similarly, for every system of entangled qubits we introduce a variable $e \in l_{E}$. The domain of $e$ is the set of names of quantum states that the system may attain, together with the value $\perp$ indicating that either the system is not in a pure state or its qubits are not entangled.

- Each variable $q$ and $e$ is assigned a name if only if they are pure and cannot be further separated. Otherwise, they are assigned the value $\perp$. The global state $\sigma$ is then the tensor product of these substates.

- In addition, we make use of an auxiliary variable $q^{\prime}$ for each qubit $q \in \mathscr{N}$ recording the name of its initial state, and introduce the global counter $g c \in l_{E}$ that increases with every action in the network. This is used to track the global time and to enumerate the configurations in $\mathscr{C}_{\mathscr{N}}$ according to their occurrence in the path. 


\begin{tabular}{|c|c|c|c|}
\hline Action & Qubit/Entangled System & State & Name \\
\hline \multirow{2}{*}{ Initially } & $q_{1}$ & {$[a, b]^{T}$} & $q s_{1}$ \\
\cline { 2 - 4 } & $e_{23}$ & $\frac{1}{2}[1,1,1,-1]^{T}$ & $q s_{2}$ \\
\hline$E_{12}$ & $e_{123}$ & $\frac{1}{2}[a, a, a,-a, b, b,-b, b]^{T}$ & $q s_{3}$ \\
\hline \multirow{4}{*}{$M_{1}^{0}$} & $q_{1}$ & $\frac{1}{2}[\sqrt{2}, \sqrt{2}]^{T}$ & $q s_{4}$ \\
& $e_{23}$ & $\frac{1}{2}[\sqrt{2},-\sqrt{2}]^{T}$ & $q s_{5}$ \\
\cline { 2 - 4 } & $q_{2}$ & $\frac{1}{2}[a+b, a+b, a-b,-a+b]^{T}$ & $q s_{6}$ \\
& & $\frac{1}{2}[a-b, a-b, a+b,-a-b]^{T}$ & $q s_{7}$ \\
\hline \multirow{4}{*}{$M_{2}^{0}$} & & $\frac{1}{2}[\sqrt{2}, \sqrt{2}]^{T}$ & $q s_{4}$ \\
& $q_{3}$ & $\frac{1}{2}[\sqrt{2},-\sqrt{2}]^{T}$ & $q s_{5}$ \\
\cline { 2 - 4 } & & {$[a, b]^{T}$} & $q s_{1}$ \\
& & {$[a,-b]^{T}$} & $q s_{8}$ \\
& $q_{3}$ & {$[b, a]^{T}$} & $q s_{9}$ \\
$X_{3}^{x_{2}} Z_{3}^{x_{1}}$ & & {$[a, b]^{T}$} & $q s_{10}$ \\
\hline
\end{tabular}

Table 1: Enumeration of quantum substates in the evolution of QTP.

Example 2. The global quantum state of QTP is represented in the local state of the Environment $E$ as the tuple $l_{E}=\left(q_{1}, q_{2}, q_{3}, q_{1}^{\prime}, q_{2}^{\prime}, q_{3}^{\prime}, e_{23}, e_{123}, g c\right)$. The initial state of the input qubit $q_{1}$ is $[a, b]^{T}$, for $a, b \in \mathbb{C}$. We assume that it is not equal to states $[1,0]^{T}$ and $[0,1]^{T}$ of the standard basis, nor to states $\frac{1}{2}[\sqrt{2}, \sqrt{2}]^{T}$ and $\frac{1}{2}[\sqrt{2},-\sqrt{2}]^{T}$ of the measurement basis. In these cases there are fewer states, but the procedure is analogous. Table 1 shows the enumeration of substates occurring in all possible runs of the network, as Alice and Bob execute quantum commands according to QTP. For instance, the initial state of the network is $\left(q s_{1}, \perp, \perp, q s_{1}, \perp, \perp, q s_{2}, \perp, 1\right)$. Note that only the input qubit $q_{1}$ and the system of two entangled qubit $e_{23}$ have assigned named states. This is because the individual qubits $q_{2}$ and $q_{3}$ are not in a pure state and the system of all three qubit $e_{123}$ can be further separated. Indeed, the whole quantum state can be expressed as the tensor product $[a, b]^{T} \otimes \frac{1}{2}[1,1,1,-1]^{T}$, or by using names $q s_{1} \otimes q s_{2}$.

\subsection{Transition Rules}

Events in the event sequence $\mathscr{E}_{i}$ of agent $\mathbf{A}_{i}$ are mapped into actions in $A c t_{i}$. Actions are executed according to a protocol function $P_{i}$ and their effects are described by evolution functions $t_{i}$ and $t_{E}$ depending on whether the classical state of agent $\mathbf{A}_{i}$ changes, or the quantum state $\sigma$ of the system changes, or both. Before introducing the mapping for events, note that DMC is a probabilistic calculus, whereas IS have a Boolean semantics. We deal with this issue by allowing all admissible transitions, abstracting away from the probability distribution. As a result, we lose the ability to reason about the probability of reaching a state. However, this is not an issue for us as we need to reason about non-probabilistic properties only as the choice of the language $\mathscr{L}$ demonstrates.

Note also that the execution of a pattern $\mathscr{P}$ in DMC occurs in a single transition step and depends on the big-step semantics of the pattern (see Rule 1). However, we handle transitions at the level of individual commands of $\mathscr{P}$, and so the execution depends on the small-step semantics of patterns and may span across several time steps. This leads to a finely grained state space. In the rest of this section we present the actions, the protocols, and the evolution functions associated with the classical and quantum communication and the quantum commands presented in Section 2.1 .

Classical rendez-vous. Assume that agent $\mathbf{A}_{i}$ sends the value of $y$ to agent $\mathbf{A}_{j}$ who stores it in $x$, specified in DMC as $\Gamma_{i}, \mathbf{A}_{i}: Q_{i} . \mathrm{c} ! y$ and $\Gamma_{j}, \mathbf{A}_{j}: Q_{j} . \mathrm{c} ? x$, and that this is the $v$ th (resp. $w$ th) event in $\mathscr{E}_{i}$ 
(resp. $\mathscr{E}_{j}$ ). We translate this by considering the actions $s n d_{-} j_{-} y_{0}$ and $s n d_{-} j_{-} y_{1}$ in the set $A c t_{i}$ of actions for agent $i$, and action $r c v_{-} i_{-} x$ in $A c t_{j}$. The protocol functions are:

$$
\begin{aligned}
P_{i}\left(l_{i}\right) & =\left\{s n d_{-} j_{-} y_{0}\right\}, \text { if } p c=v \wedge y=0, \\
P_{i}\left(l_{i}\right) & =\left\{s n d_{-} j_{-} y_{1}\right\}, \text { if } p c=v \wedge y=1, \\
P_{j}\left(l_{j}\right) & =\left\{r c v_{-} i_{-} x\right\}, \text { if } p c=w .
\end{aligned}
$$

The configuration transition, described by Rule 2 , is translated into the following evolution functions for the agents $i$ and $j$ :

$$
\begin{aligned}
t_{i}\left(l_{i}, A c t_{i}, A c t_{j}\right) & =p c \mapsto p c+1, \text { if }\left(A c t_{i}=s n d_{-} j_{-} y_{0} \vee A c t_{i}=s n d_{-} j_{-} y_{1}\right) \wedge A c t_{j}=r c v i_{-} x, \\
t_{j}\left(l_{j}, A c t_{i}, A c t_{j}\right) & =p c \mapsto p c+1 \wedge x \mapsto 0, \text { if } A c t_{i}=s n d_{-} j_{-} y_{0} \wedge A c t_{j}=r c v \_i-x, \\
t_{j}\left(l_{j}, A c t_{i}, A c t_{j}\right) & =p c \mapsto p c+1 \wedge x \mapsto 1, \text { if } A c t_{i}=s n d_{-} j_{-} y_{1} \wedge A c t_{j}=r c v \_i \_x .
\end{aligned}
$$

The rationale behind the above equations is that when agents perform paired send/receive actions at the same time step, their program counters are incremented, and variable $x$ of agent $\mathbf{A}_{j}$ is assigned the transmitted value.

Quantum communication. Assume that agent $\mathbf{A}_{i}$ sends a qubit $q \in Q_{i}$ to agent $\mathbf{A}_{j}$, described as $\Gamma_{i}, \mathbf{A}_{i}: Q_{i} . \mathrm{qc} ! q$ and $\Gamma_{j}, \mathbf{A}_{j}: Q_{j} . \mathrm{qc} ? q$, and that this is the $v$ th (resp. $w$ th) event in $\mathscr{E}_{i}\left(\right.$ resp. $\mathscr{E}_{j}$ ). We introduce actions $q s n d_{-} j_{-} q$ and $q r c v i_{-} q$ in $A c t_{i}$ and $A c t_{j}$ respectively. The protocol functions are:

$$
\begin{aligned}
P_{i}\left(l_{i}\right) & =\left\{q s n d_{-} j_{-} q\right\}, \text { if } p c=v, \\
P_{j}\left(l_{j}\right) & =\left\{q r c v_{-} i_{-}\right\}, \text {if } p c=w .
\end{aligned}
$$

Rule 3 defines the configuration transition in terms of sets of qubits $Q_{i}$ and $Q_{j}$. When $\mathbf{A}_{i}$ sends the qubit $q$, it is removed from her set, and when $\mathbf{A}_{j}$ receives $q$, it is added to her set. This is translated into IS by the evolution functions:

$$
\begin{aligned}
t_{i}\left(l_{i}, A c t_{i}, A c t_{j}\right) & =p c \mapsto p c+1 \wedge q \mapsto 0, \text { if } A c t_{i}=q s n d_{-} j_{-} q \wedge A c t_{j}=q r c v_{-} i_{-} q, \\
t_{j}\left(l_{j}, A c t_{i}, A c t_{j}\right) & =p c \mapsto p c+1 \wedge q \mapsto 1, \text { if } A_{c t}=q s n d_{-} j_{-} q \wedge A c t_{j}=q r c v_{-} i_{-} q .
\end{aligned}
$$

This means that when both agents concurrently execute the respective quantum communication events, their local program counters are incremented, and the ownership of the qubit changes, i.e., $\mathbf{A}_{i}$ is no longer in possession of $q$ while $\mathbf{A}_{j}$ owns it but does not know its state.

Corrections. The events $X_{q}^{s}$ and $Z_{q}^{s}$ differ only in their matrix representations, so we describe them together. Assume that agent $\mathbf{A}_{i}$ executes the Pauli operator $X$ or the Pauli operator $Z$ on a qubit $q$ at step $v$ of $\mathscr{E}_{i}$ if signal $s=1$, otherwise she skips the event. This scenario has the following DMC description: $\Gamma_{i}, \mathbf{A}_{i}: q \uplus R_{i} . U_{q}^{s}$, with $U_{q}^{s} \in\left\{X_{q}^{s}, Z_{q}^{s}\right\}$. We introduce actions $x_{-} q$ and $z_{-} q$ in $A c t_{i}$, and since the agent applies the event conditionally, we also include the action skip. In the rest of the description we refer to both actions $x_{-} q$ and $z_{-} q$ as $u_{-} q$. The protocol function is then given as:

$$
\begin{array}{ll}
P_{i}\left(l_{i}\right)=\{\text { skip }\} & \text { if } p c=v \wedge s=0 ; \\
P_{i}\left(l_{i}\right)=\left\{u_{-q}\right\} & \text { if } p c=v \wedge s=1 .
\end{array}
$$

For example, we have the following ground protocol function for Bob in QTP:

$$
\begin{array}{ll}
P_{B}\left(l_{B}\right)=\{s k i p\}, \text { if } p c=3 \wedge x_{1}=0 ; & P_{B}\left(l_{B}\right)=\{s k i p\}, \text { if } p c=4 \wedge x_{2}=0 ; \\
P_{B}\left(l_{B}\right)=\left\{z_{-} q_{3}\right\}, \text { if } p c=3 \wedge x_{1}=1 ; & P_{B}\left(l_{B}\right)=\left\{x_{-} q_{3}\right\}, \text { if } p c=4 \wedge x_{2}=1 .
\end{array}
$$


The small-step semantics for corrections is defined as $\sigma, \Gamma_{i} \stackrel{U_{q}^{s}}{\longrightarrow} U_{r}^{s_{\Gamma_{i}}} \sigma, \Gamma_{i}$. Assume that the qubit $q$ is in system $e$, which again may be just $q$ or some entangled system. The local state of the agent $\mathbf{A}_{i}$ changes only through the $p c$ increment. We define the evolution functions as:

$$
\begin{aligned}
t_{E}\left(l_{E}, A c t_{i}\right) & =g c \mapsto g c+1 \wedge e \mapsto q s_{y}, \text { if } e=q s_{x} \wedge A c t_{i}=u_{-} q ; \\
t_{i}\left(l_{i}, A c t_{i}\right) & =p c \mapsto p c+1, \text { if } A c t_{i}=u_{-} q \vee A c t_{i}=s k i p ;
\end{aligned}
$$

where $q s_{x}$ (resp. $q s_{y}$ ) is the name of the state before (resp. after) the execution. The ground evolution function of $E$ in QTP with respect to Bob's corrections $X_{3}^{x_{2}} Z_{3}^{x_{1}}$ is given as the following equations corresponding to measurement outcomes $x_{1} x_{2} \mapsto 10, x_{1} x_{2} \mapsto 01$, and $x_{1} x_{2} \mapsto 11$ respectively. Note that in the last case Bob executes both actions $z_{-} q_{3}$ and $x_{-} q_{3}$ sequentially, while in the first two cases he executes only one of them and skips the other.

$$
\begin{aligned}
& t_{E}\left(l_{E}, A c t_{B}\right)=g c \mapsto g c+1 \wedge q_{3} \mapsto q s_{1}, \text { if } q_{3}=q s_{8} \wedge A c t_{B}=z_{-} q_{3} ; \\
& t_{E}\left(l_{E}, A c t_{B}\right)=g c \mapsto g c+1 \wedge q_{3} \mapsto q s_{1}, \text { if } q_{3}=q s_{9} \wedge A c t_{B}=x_{-} q_{3} ; \\
& t_{E}\left(l_{E}, A c t_{B}\right)=g c \mapsto g c+1 \wedge q_{3} \mapsto q s_{9}, \text { if } q_{3}=q s_{10} \wedge A c t_{B}=z_{-} q_{3} .
\end{aligned}
$$

Entanglement. Assume that agent $\mathbf{A}_{i}$ applies at step $v$ of $\mathscr{E}_{i}$ the entanglement operator $E_{q r}$ on qubits $q$ and $r$. The DMC definition of the agent in this case is $\Gamma_{i}, \mathbf{A}_{i}: q, r \uplus R_{i} . E_{q r}$. Since this event is independent of signals, we add only one corresponding action $e n t_{-} q_{-} r$ to $A c t_{i}$ and define the following protocol function: $P_{i}\left(l_{i}\right)=\{$ ent_q_ $r\}$, if $p c=v$. The small-step rule for entanglement is given as $\sigma, \Gamma_{i} \stackrel{E_{q r}}{\longrightarrow}{ }^{C} Z_{q r} \sigma, \Gamma_{i}$, where ${ }^{C} Z_{q r}$ is the controlled- $Z$ operator realising the entanglement. Since we divide the global state $\sigma$ into its smallest pure substates, we have two possible situations. In the first case $q \in e^{\prime}$ and $r \in e^{\prime \prime}$, where $e^{\prime}$ and $e^{\prime \prime}$ are isolated qubits, distinct entangled systems, or combination of both. The resulting entangled system $e$ is the union of the two systems $e^{\prime}$ and $e^{\prime \prime}$, and we define the evolution function of the Environment $E$ as:

$$
t_{E}\left(l_{E}, A c t_{i}\right)=g c \mapsto g c+1 \wedge e \mapsto q s_{z} \wedge e^{\prime} \mapsto \perp \wedge e^{\prime \prime} \mapsto \perp \text {, if } e^{\prime}=q c_{x} \wedge e^{\prime \prime}=q c_{y} \wedge A c t_{i}=e n t_{-} q_{-} r ;
$$

where $q s_{x}, q s_{y}$, and $q s_{z}$ are the names of the quantum states in which the systems $e^{\prime}, e^{\prime \prime}$ are during the execution of the event, and $e$ after the execution. For instance, the ground evolution function in QTP for Alice's entanglement $E_{12}$ is:

$$
\begin{aligned}
t_{E}\left(l_{E}, A c t_{A}\right)=g c \mapsto g c+1 & \wedge e_{123} \mapsto q s_{3} \wedge q_{1} \mapsto \perp \wedge e_{23} \mapsto \perp, \\
& \text { if } q_{1}=q c_{1} \wedge e_{23}=q c_{2} \wedge A c t_{A}=\text { ent_ } q_{1-q_{2}} .
\end{aligned}
$$

Note that there may be many possible combinations of various states for $e^{\prime}$ and $e^{\prime \prime}$, and we have to define the evolution function for all of them. In the second case the qubits $q$ and $r$ are part of the same system $e$ and we simply have the evolution function:

$$
t_{E}\left(l_{E}, A c t_{i}\right)=g c \mapsto g c+1 \wedge e \mapsto q s_{y}, \text { if } e=q s_{x} \wedge A c t_{i}=e n t_{-} q_{-} r
$$

where $q s_{x}$ (resp. $q s_{y}$ ) is the name of the state before (resp. after) the execution. In both cases the local state of agent $\mathbf{A}_{i}$ is updated as follows:

$$
t_{i}\left(l_{i}, A c t_{i}\right)=p c \mapsto p c+1 \wedge q \mapsto 1 \wedge r \mapsto 1, \text { if } A c t_{i}=e n t_{-} q_{-} r .
$$

This equation states that the counter of $\mathbf{A}_{i}$ is incremented and the agent loses any knowledge about the state of $q$ and $r$ she might have had, since neither qubit is in a pure state anymore.

Measurement. This is a complex event modifying the quantum state of the network as well as the local states of agents. Suppose that agent $\mathbf{A}_{i}$ in step $v$ of $\mathscr{E}_{i}$ measures her qubit $q$ in the $\{|+\alpha\rangle,|-\alpha\rangle\}$ basis, specified in DMC as $\Gamma_{i}, \mathbf{A}_{i}: q \uplus R_{i} \cdot{ }^{t}\left[M_{q}^{\alpha}\right]^{s}$, where $s$ and $t$ are signals. A measurement is a stochastic event 


\begin{tabular}{|c|c|c|}
\hline \multirow{2}{*}{$\emptyset \emptyset$} & Actions & $m_{-} q_{-}+\alpha, m_{-} q_{-}-\alpha$ \\
\hline & Protocol & $P_{i}\left(l_{i}(g)\right)=\left\{m_{-} q_{-}+\alpha, m_{-} q_{-}-\alpha\right\}$, if $p c=v$ \\
\hline \multirow[b]{2}{*}{$s \emptyset$} & Actions & $m_{-} q_{-} s_{0-}+\alpha, m_{-} q_{-} s_{0_{-}-}{ }_{\alpha}, m_{-} q_{-} s_{1-}+\alpha, m_{-} q_{-} s_{1-}-\alpha$ \\
\hline & Protocol & $\begin{array}{l}P_{i}\left(l_{i}(g)\right)=\left\{m_{-} q_{-} s_{0-}+\alpha, m_{-} q_{-} s_{0-}-\alpha\right\}, \text { if } p c=v \wedge s=0 \\
P_{i}\left(l_{i}(g)\right)=\left\{m_{-} q_{-} s_{1-}+\alpha, m_{-} q_{-} s_{1-}-\alpha\right\}, \text { if } p c=v \wedge s=1\end{array}$ \\
\hline \multirow[b]{2}{*}{$\emptyset t$} & Actions & $m_{-} q_{-} t_{0-}+\alpha, m_{-} q_{-} t_{0-}-\alpha, m_{-} q_{-} t_{1-}+\alpha, m_{-} q_{-} t_{1-}-\alpha$ \\
\hline & Protocol & $\begin{array}{l}P_{i}\left(l_{i}(g)\right)=\left\{m_{-} q_{-} t_{0-}+\alpha, m_{-} q_{-} t_{0-}-\alpha\right\}, \text { if } p c=v \wedge t=0 \\
P_{i}\left(l_{i}(g)\right)=\left\{m_{-} q_{-} t_{1-}+\alpha, m_{-} q_{-} t_{1-}-\alpha\right\}, \text { if } p c=v \wedge t=1\end{array}$ \\
\hline \multirow[b]{2}{*}{$s t$} & Actions & $\begin{array}{l}m_{-} q_{-} s_{0 \_} t_{0-}+\alpha, m_{-} q_{-} s_{0 \_} t_{0-}-\alpha, m_{-} q_{-} s_{0 \_} t_{1-}+\alpha, m_{-} q_{-} s_{0} t_{1-}-\alpha \\
m_{-} q_{-} s_{1} t_{0-}+\alpha, m_{-} q_{-} s_{1} t_{0-}-\alpha, m_{-} q_{-} s_{1} t_{1-}+\alpha, m_{-} q_{-} s_{1} t_{1-}-\alpha\end{array}$ \\
\hline & Protocol & $\begin{array}{l}P_{i}\left(l_{i}(g)\right)=\left\{m_{-} q_{-} s_{0} t_{0-}+\alpha, m_{-} q_{-} s_{0} t_{0-}-\alpha\right\}, \text { if } p c=v \wedge s=0 \wedge t=0 \\
P_{i}\left(l_{i}(g)\right)=\left\{m_{-} q_{-} s_{0} t_{1-}+\alpha, m_{-} q_{-} s_{0} t_{1-}-\alpha\right\}, \text { if } p c=v \wedge s=0 \wedge t=1 \\
P_{i}\left(l_{i}(g)\right)=\left\{m_{-} q_{-} s_{1-} t_{0-}+\alpha, m_{-} q_{-} s_{1} t_{0-}-\alpha\right\}, \text { if } p c=v \wedge s=1 \wedge t=0 \\
P_{i}\left(l_{i}(g)\right)=\left\{m_{-} q_{-} s_{1} t_{1-}+\alpha, m_{-} q_{-} s_{1} t_{1-}-\alpha\right\}, \text { if } p c=v \wedge s=1 \wedge t=1\end{array}$ \\
\hline
\end{tabular}

Table 2: Actions and protocol rules for various degree of dependency of measurements.

and may also depend on signals $s$ and $t$. We express this non-determinism by associating two actions to a given local state $l_{i}$ of agent $i$. However, due to a possible dependency on signals $s$ and $t$, there are four different sets of actions and protocol rules. We list them in Table 2, where $\emptyset$ means that the measurement does not depend on a particular signal.

The following two transitions are defined in the small-step semantics for the measurement event: $\sigma, \Gamma_{i} \stackrel{{ }^{t}\left[M_{q}^{\alpha}\right]^{r}}{\longrightarrow} \lambda\left\langle+\left.\alpha_{\Gamma_{i}}\right|_{q} \sigma, \Gamma_{i}[0 / q]\right.$ and $\sigma, \Gamma_{i} \stackrel{{ }^{t}\left[M_{r}^{\alpha}\right]^{s_{1}}}{\longrightarrow} \lambda\left\langle-\left.\alpha_{\Gamma_{i}}\right|_{q} \sigma, \Gamma_{i}[1 / q]\right.$. This is the source of non-determinism in the transition system, but we do not consider the probability $\lambda$ as long as it is non-zero.

There are again four types of evolution functions. They differ in the computation of quantum states, and since we give only the general rules, here we describe the evolution functions only for the independent measurement, i.e., when $s=t=\emptyset$. As far as the translation rules are concerned, the other three types differ only in the names of the actions and the actual names of quantum states. We can translate them analogously.

We now consider two cases where both measurement outcome are possible. First, for the measurement of an isolated qubit $q$ we define the evolution function of the Environment $E$ as follows:

$$
\begin{aligned}
& t_{E}\left(l_{E}, A c t_{i}\right)=g c \mapsto g c+1 \wedge q \mapsto q s_{+_{\alpha}}, \text { if } q=q c_{x} \wedge A c t_{i}=m_{-} q_{-}+\alpha ; \\
& t_{E}\left(l_{E}, A c t_{i}\right)=g c \mapsto g c+1 \wedge q \mapsto q s_{-_{\alpha}}, \text { if } q=q c_{x} \wedge A c t_{i}=m_{-} q_{-}-\alpha ;
\end{aligned}
$$

where $q s_{+_{\alpha}}$ and $q s_{-\alpha}$ are names of the $\left\{\left|+_{\alpha}\right\rangle,\left|{ }_{\alpha}\right\rangle\right\}$ measurement basis. If the qubit $q$ is part of an entangled system $e$, then the system becomes separated on measurement. The measured qubit $q$ collapses and the rest of qubits form a new system $e^{\prime}$. We define the evolution function as follows:

$$
\begin{aligned}
& t_{E}\left(l_{E}, A c t_{i}\right)=g c \mapsto g c+1 \wedge q \mapsto q s_{+\alpha} \wedge e \mapsto \perp \wedge e^{\prime} \mapsto q s_{y}, \text { if } e=q c_{x} \wedge A c t_{i}=m_{-} q_{-}+\alpha, \\
& t_{E}\left(l_{E}, A c t_{i}\right)=g c \mapsto g c+1 \wedge q \mapsto q s_{-\alpha} \wedge e \mapsto \perp \wedge e^{\prime} \mapsto q s_{z}, \text { if } e=q c_{x} \wedge A c t_{i}=m_{-} q_{-}-\alpha .
\end{aligned}
$$

In both cases the measurement outcome is assigned to a signal variable $s^{\prime}$ of agent $\mathbf{A}_{i}$ and her evolution function is given by:

$$
\begin{aligned}
& t_{i}\left(l_{i}, A c t_{i}\right)=p c \mapsto p c+1 \wedge s^{\prime} \mapsto 0 \wedge q \mapsto 2, \text { if } A c t_{i}=m_{-} q_{-}+\alpha \\
& t_{i}\left(l_{i}, A c t_{i}\right)=p c \mapsto p c+1 \wedge s^{\prime} \mapsto 1 \wedge q \mapsto 2, \text { if } \text { Act }_{i}=m_{-} q_{-}-\alpha .
\end{aligned}
$$

For instance, consider the first measurement that Alice performs in QTP. All three qubits are entangled together and therefore measuring the input qubit $q_{1}$ causes separation of the system $e_{123}$ into two parts, 
$q_{1}$ and $e_{23}$, and has two possible outcomes. Both have probability $\lambda=0.5$, but we do not take this into account since all we require is that they are non-zero, therefore the respective transitions are admissible. We have the following ground evolution functions for the Environment and Alice:

$$
\begin{aligned}
& t_{E}\left(l_{E}, A c t_{A}\right)=g c \mapsto g c+1 \wedge q_{1} \mapsto q s_{4} \wedge e_{123} \mapsto \perp \wedge e_{23} \mapsto q s_{6}, \text { if } e_{123}=q c_{3} \wedge A c t_{A}=m_{-} q_{1-}+\alpha ; \\
& t_{E}\left(l_{E}, A c t_{A}\right)=g c \mapsto g c+1 \wedge q_{1} \mapsto q s_{5} \wedge e_{123} \mapsto \perp \wedge e_{23} \mapsto q s_{7}, \text { if } e_{123}=q c_{3} \wedge A c t_{A}=m_{-} q_{1-}-\alpha ; \\
& t_{A}\left(l_{A}, A c t_{A}\right)=p c \mapsto p c+1 \wedge s_{1} \mapsto 0 \wedge q_{1} \mapsto 2, \text { if } A_{c t_{A}}=m_{-} q_{1-}+\alpha ; \\
& t_{A}\left(l_{A}, A c t_{A}\right)=p c \mapsto p c+1 \wedge s_{1} \mapsto 1 \wedge q_{1} \mapsto 2, \text { if } A_{c t_{A}}=m_{-} q_{1-}-\alpha .
\end{aligned}
$$

In the case that the measured qubit is in a state that coincides with one of the states of the measurement basis, there is only one possible outcome and we need to prevent reaching an impossible state. The translation of the transition function in case that a measurement outcome has zero probability requires modification of the evolution functions. We only show the case when measuring $\left|-{ }_{\alpha}\right\rangle$ is impossible. The evolution function of the Environment is given as:

$$
t_{E}\left(l_{E}, A c t_{i}\right)=g c \mapsto g c+1 \wedge q \mapsto q s_{+\alpha}, \text { if } q=q c_{+\alpha} \wedge\left(A c t_{i}=m_{-} q_{-}+_{\alpha} \vee A c t_{i}=m_{-} q_{-}-\alpha\right) .
$$

The Environment "signals" that the measurement of $q$ in a quantum state $q s_{x}$ has only one possible outcome. We introduce action $e n v_{x}$ in $A c t_{E}$ and define the following protocol function: $P_{E}\left(l_{E}\right)=\left\{e n v_{x}\right\}$, if $q=q s_{x}$. The evolution function of agent $i$ is then defined as:

$$
\begin{aligned}
& t_{i}\left(l_{i}, A c t_{i}, A c t_{E}\right)=p c \mapsto p c+1 \wedge s^{\prime} \mapsto 0 \wedge q \mapsto 2, \text { if } A c t_{i}=m_{-} q_{-}+\alpha \vee\left(A c t_{i}=m_{-} q_{-}-\alpha \wedge A c t_{E}=e n v_{x}\right), \\
& t_{i}\left(l_{i}, A c t_{i}, A c t_{E}\right)=p c \mapsto p c+1 \wedge s^{\prime} \mapsto 1 \wedge q \mapsto 2, \text { if } A c t_{i}=m_{-} q_{-}-\alpha \wedge A c t_{E} \neq e n v_{x} .
\end{aligned}
$$

\subsection{Correctness Proof}

We now show that the translation $f$ defined in the previous section is sound, that is, $f$ preserves the truth conditions of formulae defined in the language $\mathscr{L}$ introduced in Section 2.2 from the set of atomic propositions $A P=\left\{x=y, q_{i}=q_{j}\right\}$. In [7] the truth conditions for the atoms in $A P$ in a configuration $C$ of a network $\mathscr{N}$ are given as follows:

$$
\begin{gathered}
(C, \mathscr{N}) \models x=y \quad \text { iff } \quad \text { there are agents } i, j \text { such that } \Gamma_{i}(x)=\Gamma_{i}(y) ; \\
(C, \mathscr{N}) \models q_{i}=q_{j} \quad \text { iff } \quad \text { the global quantum state } \sigma \text { is such that } \sigma=q_{i}=q_{j} .
\end{gathered}
$$

Intuitively, $x=y$ holds iff the bits denoted by $x$ and $y$ are equal. Also, $q_{i}=q_{j}$ holds iff the qubits denoted by $q_{i}$ and $q_{j}$ are equal. We can prove the following result on the translation $f$ and the language $\mathscr{L}$.

Theorem 1. For every formula $\phi \in \mathscr{L}$,

$$
(C, \mathscr{N}) \models \phi \quad \text { iff } \quad(f(\mathscr{N}), f(C)) \models \phi
$$

Proof. The proof is by induction on the length of $\phi$. For reasons of space, we only provide a sketch of the proof. If $\phi$ is an atomic formula, then $\phi$ is of the form $\mathfrak{a}=\mathfrak{b}$, where $\mathfrak{a}$ and $\mathfrak{b}$ are both either bits or qubits. By the definition of $f(C)$ in Section 3.1 we can easily check that $(C, \mathscr{N}) \models \mathfrak{a}=\mathfrak{b}$ iff $(f(\mathscr{N}), f(C)) \models \mathfrak{a}=\mathfrak{b}$. Thus, the base case holds. The inductive case for propositional connectives $\neg$ and $\vee$ is straightforward.

If $\phi=E X \psi$, then by the translation of events in the event sequence $\mathscr{E}$ into actions in Act defined in Section 3.2, we can see that two configurations $C, C^{\prime} \in \mathscr{N}$ are in the temporal relation induced by 
$\mathscr{E}$, or $C \stackrel{\mathscr{N}}{\longrightarrow} C^{\prime}$, iff their translations $f(C), f\left(C^{\prime}\right) \in f(\mathscr{N})$ are in the temporal relation induced by $A c t$, or $f(C) \stackrel{f(\mathscr{N})}{\longrightarrow} f\left(C^{\prime}\right)$. The result then follows by the induction hypothesis. The inductive case for the other temporal operators is similar.

If $\phi=K_{i} \psi$, then by the definition of the local state $l_{i}$ of an agent $i$ in Section 3.1, we have that $l_{i}(f(C))=l_{i}^{\prime}\left(f\left(C^{\prime}\right)\right)$ iff $\Gamma_{i}=\Gamma_{i}^{\prime}$ and $\mathscr{E}_{i}=\mathscr{E}_{i}^{\prime}$, that is, $C \sim_{i}^{\mathscr{N}} C^{\prime}$ iff $f(C) \sim_{i}^{f(\mathscr{N})} f\left(C^{\prime}\right)$. Also in this case the result follows by the induction hypothesis. This completes the sketch.

Theorem 1 allows us to check whether a specification $\phi \in \mathscr{L}$ is satisfied in a network $\mathscr{N}$ by verifying $\phi$ in the corresponding interpreted system $f(\mathscr{N})$.

\section{Implementation and Evaluation}

In this section we present an implementation of the formal map above. DMC2ISPL 1 is a source-to-source compiler, written in C++ and using GNU Octave libraries for matrix operations. DMC2ISPL translates a protocol specified in a machine-readable DMC input format into an ISPL program. The code generated is then run by MCMAS, which in turn reports on the specification requirements of the protocol.

We modified DMC, so it can be read by the compiler. The adaptation closely follows the syntax of the original DMC, but also reflects some features of ISPL. A DMC file consists of five modules: a set of agents, a set of qubits, whose initial state is explicitly declared, a set of groups of agents that are used in formulae involving group modalities, a set of formulae to be verified, and a set of macros that allow agents to perform complex quantum operations in a single time step. The declaration of an agent consists of a set of input qubits, a set of a priori known qubits, a set of classical inputs, and a set of events the agent executes. For illustration, the DMC code snippet for QTP can be found in Listing 1.

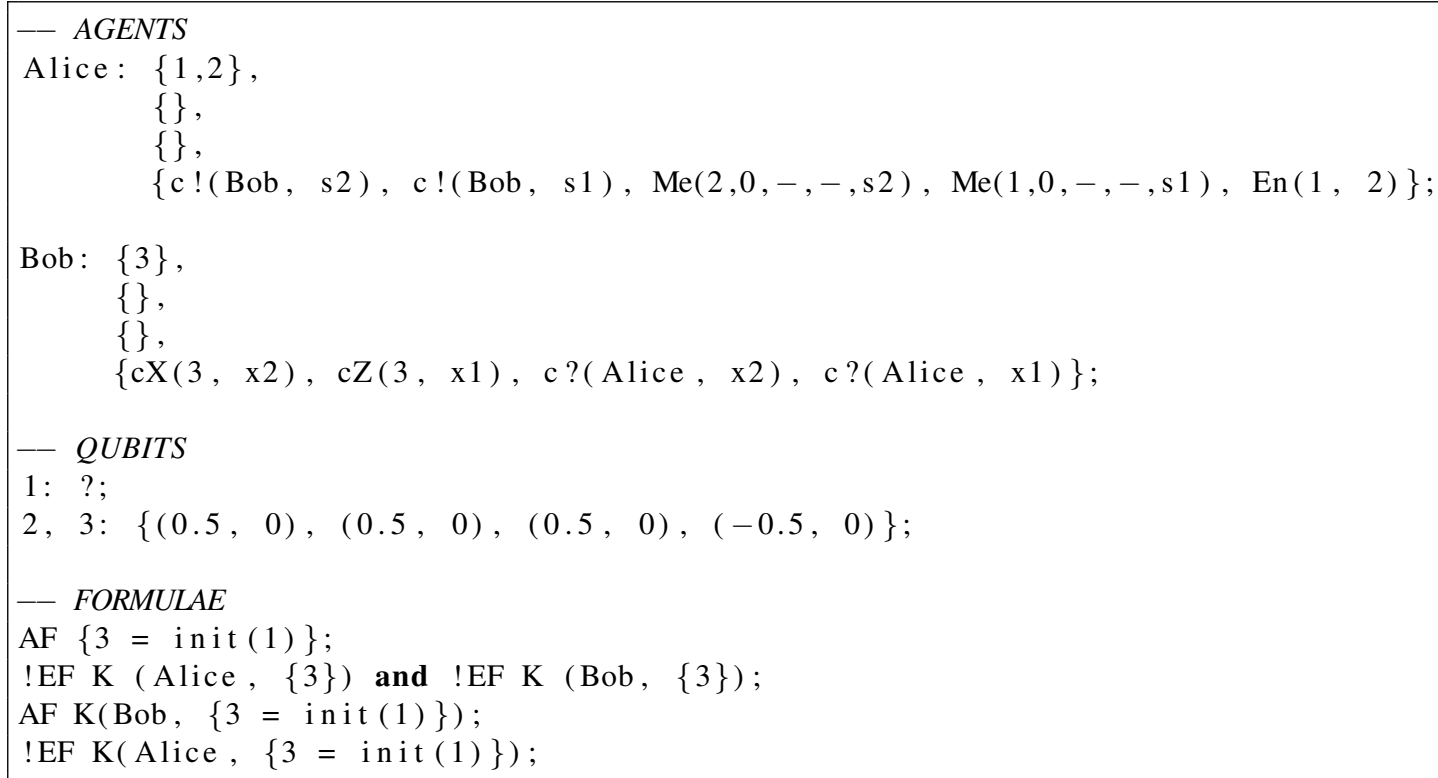

Listing 1: QTP.dmc

DMC2ISPL has the architecture of a standard compiler. It consists of the three following components: a module for parsing and validating the DMC input file, a module for generating the reachable quantum

${ }^{1}$ The source code is available from http://www.doc.ic.ac.uk/ pg809/dmc2ispl.tar.gz 


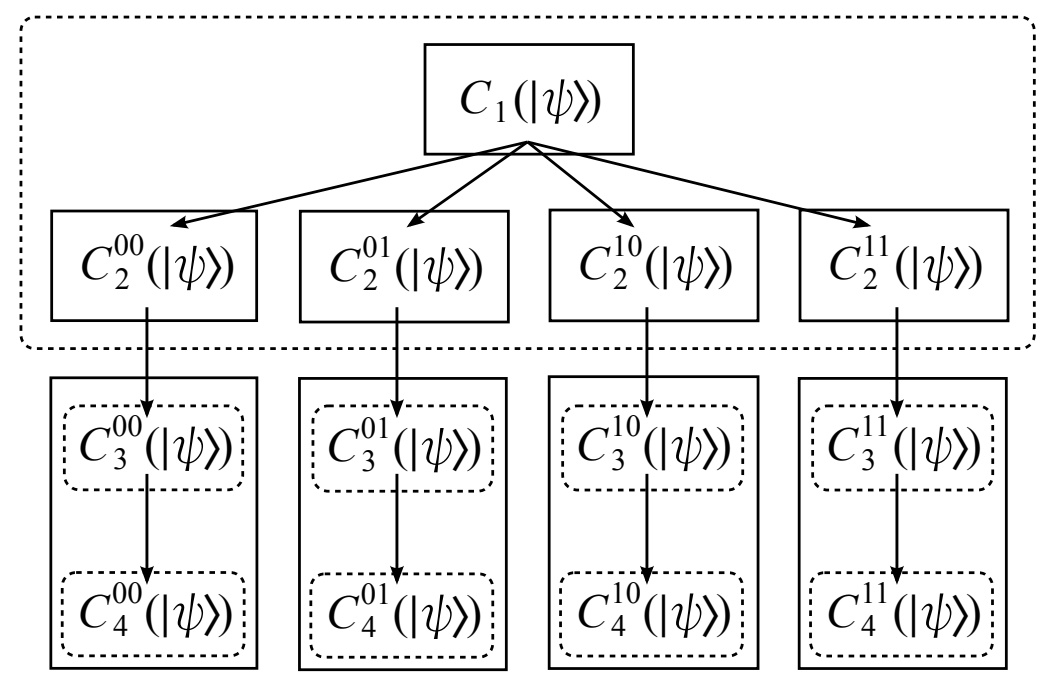

Figure 1: The epistemic accessibility relations of Alice and Bob in the QTP network.

state space, and a module for generating the ISPL output file. Essentially, since MCMAS does not support matrix arithmetic, the compiler is responsible for computation of the reachable quantum state space, enumeration of encountered quantum states, and generation of the evolution function of the global quantum system. Quantum states of a $n$-qubit system are represented as $2^{n} \times 1$ complex matrices and unitary operators and measurement projections as $2^{n} \times 2^{n}$ sparse complex matrices. After the elimination of the global phase, whenever two identical state matrices are encountered during the evolution of the quantum state of the $n$-qubit system, they have assigned the same name. MCMAS then works with these enumerations.

We used the compiler to verify QTP, as well as the Quantum Key Distribution (QKD) [12], and the Superdense Coding (SDC) [4] protocol against the properties from the reference papers [7, 10]. Table 3 summarises these properties. We discuss QTP in more detail.

The figure 1 gives a graphical representation of the possible configurations in the QTP network. Note that configurations are parametrised by measurement outcomes and the quantum input $|\psi\rangle$. The first formula in QTP section of Table 3 states that the $\mathscr{N}_{T P}$ network is correct, since the state of Bob's qubit $q_{3}$ will eventually be equal to the initial state of Alice's qubit $q_{1}$. The second formula states that neither agent knows the actual quantum state of the qubit $q_{3}$ at any point of the computation. The third formula states that Bob eventually knows that the state of his qubit $q_{3}$ is equal to the initial state of qubit $q_{1}$. The last formula states that Alice never knows this fact.

Interestingly, while [10] states that all four formulae are true in the model, MCMAS evaluated the last formula to false. The reason is that even though Alice cannot distinguish configuration $C_{3}^{00}(|\psi\rangle)$ from $C_{4}^{00}(|\psi\rangle)$, the atom $q_{3}=\operatorname{init}\left(q_{1}\right)$ holds in both configurations as Bob does not apply any correction for measurement outcomes $s_{1} s_{2} \mapsto 00$, and so the quantum state of the system is invariant along this path. This shows the importance of an automated algorithmic approach to verification as opposed to a hand-made inspection.

We conclude with some performance considerations. The tests were carried out on a 32-bit Fedora 12 Linux machine with a $2.26 \mathrm{GHz}$ Intel Core2 Duo processor and 2.9GiB RAM as follows: first, we translated the DMC specification into the corresponding ISPL code using the compiler, then we analysed the resulting code using MCMAS. Table 4 reports the results for the three protocols. It can be seen that 


\begin{tabular}{|c|c|c|}
\hline Protocol & Formula & Reading \\
\hline \multirow{4}{*}{ QTP } & $A F\left(q_{3}=\operatorname{init}\left(q_{1}\right)\right)$ & $q_{3}$ eventually equals to initial $q_{1}$ \\
\cline { 2 - 3 } & $\neg E F K_{\mathbf{A}}\left(q_{3}=|\psi\rangle\right) \wedge \neg E F K_{\mathbf{B}}\left(q_{3}=|\psi\rangle\right)$ & neither A nor $\mathbf{B}$ ever knows state of $q_{3}$ \\
\cline { 2 - 3 } & $A F K_{\mathbf{B}}\left(q_{3}=\operatorname{init}\left(q_{1}\right)\right)$ & B eventually knows $q_{1}$ was teleported \\
\cline { 2 - 3 } & $\neg E F K_{\mathbf{A}}\left(q_{3}=\operatorname{init}\left(q_{1}\right)\right)$ & A never knows $q_{1}$ was teleported \\
\hline \multirow{2}{*}{ QKD } & $\alpha_{A}=\alpha_{B} \rightarrow A F\left(K_{\mathbf{A}}\left(s_{1}=s_{2}\right) \wedge K_{\mathbf{B}}\left(s_{1}=s_{2}\right)\right)$ & success if A $\& \mathbf{B}$ used the same basis \\
\cline { 2 - 3 } & $\alpha_{A} \neq \alpha_{B} \rightarrow \neg E F\left(K_{\mathbf{A}}\left(s_{1}=s_{2}\right) \vee K_{\mathbf{B}}\left(s_{1}=s_{2}\right)\right)$ & failure if $\mathbf{A} \& \mathbf{B}$ used different bases \\
\hline \multirow{3}{*}{ SDC } & $A F\left(s_{1}=y_{1} \wedge s_{2}=y_{2}\right)$ & B eventually receives the inputs of $\mathbf{A}$ \\
\cline { 2 - 3 } & $A F K_{\mathbf{B}}\left(s_{1}=y_{1} \wedge s_{2}=y_{2}\right)$ & B eventually knows the inputs \\
\cline { 2 - 3 } & $\neg E F K_{\mathbf{A}} K_{\mathbf{B}}\left(s_{1}=y_{1} \wedge s_{2}=y_{2}\right)$ & A never knows the fact above \\
\hline
\end{tabular}

Table 3: Verified properties of QKD and SDC protocols.

\begin{tabular}{|c|c|c|c|c|c|c|}
\hline \multirow{2}{*}{ Protocol } & \multicolumn{2}{|c|}{ Reachable States } & \multicolumn{2}{c|}{ Memory (kB) } & \multicolumn{2}{c|}{ Time (s) } \\
\cline { 2 - 7 } & DMC2ISPL & MCMAS & DMC2ISPL & MCMAS & DMC2ISPL & MCMAS \\
\hline QTP & 40 & 108 & 7184 & 6068 & 0.015 & 0.066 \\
QKD & 53 & 348 & 7240 & 6119 & 0.016 & 0.014 \\
SDC & 4239 & 2192 & 8132 & 6279 & 0.112 & 0.407 \\
\hline
\end{tabular}

Table 4: Verification results for QTP, QKD and SDC protocols.

all protocols were verified very quickly. This is due to their small state space and the limited number of entangled qubits involved.

However, the amount of required resources grows exponentially for a constant increase in the number of entangled qubits. Additionally, measuring a quantum system using many different measurement angles results in many unique quantum states, which in turn requires a large number of enumeration values and an extensive evolution function. This affects the verification of a quantum protocol by MCMAS. We analysed several experimental protocols to test the limits of the tool. The results showed that protocols with up to $10^{7}$ reachable classical states and 20 entangled qubits can be realistically verified.

\section{Conclusion}

In this paper we presented a methodology for the automated verification of quantum distributed systems via model checking. We defined a translation from DMC to IS, so that MCMAS can be used to verify protocols specified in DMC. Even though the translation does not take into account stochastic properties of quantum protocols, in the sense that we abstract away from the underlying probability distribution, many useful non-probabilistic properties can still be verified as shown in reference papers [7, 10]. We implemented the methodology in a source-to-source compiler and adapted the DMC formalism to be used as an input language for the compiler. Several quantum protocols were translated and their temporal epistemic properties were successfully checked with MCMAS.

Given the universality of the underlying Measurement Calculus [8], the expressive power of DMC in terms of available quantum operations is complete. However, DMC does not support any control flow statement for the classical part of protocols. This is one of the two major limitations of the technique, although it can be solved by a suitable extension of the language. Another limitation results from the state space explosion and cannot be easily overcome since the quantum simulator requires exponential time and space on a classical computer. 


\section{References}

[1] A. Baltag \& S. Smets (2005): Complete Axiomatizations for Quantum Actions. International Journal of Theoretical Physics 44(12), doi $10.1007 / \mathrm{s} 10773-005-8022-2$

[2] A. Baltag \& S. Smets (2006): LQP: the dynamic logic of quantum information. Mathematical Structures in Computer Science 16(3), doi:10.1017/S0960129506005299.

[3] A. Baltag \& S. Smets (2008): A Dynamic-Logical Perspective on Quantum Behavior. Studia Logica 89(2), doi: $10.1007 / \mathrm{s} 11225-008-9126-5$

[4] C. H. Bennett \& S. J. Wiesner (1992): Communication via one- and two-particle operators on EinsteinPodolsky-Rosen states. Physical Review Letters 69(20), doi:10.1103/PhysRevLett.69.2881.

[5] C. H. Bennett et al. (1993): Teleporting an unknown quantum state via dual classical and Einstein-PodolskyRosen channels. Physical Review Letters 70(13), doi:10.1103/PhysRevLett.70.1895

[6] E. M. Clarke, Jr., O. Grumberg \& D. A. Peled (1999): Model Checking. MIT Press.

[7] V. Danos \& E. D'Hondt (2008): Classical Knowledge for Quantum Cryptographic Reasoning. Electronic Notes in Theoretical Computer Science 192(3), doi:10.1016/j.entcs.2008.10.026.

[8] V. Danos, E. Kashefi \& P. Panangaden (2007): The Measurement Calculus. J. ACM 54(2), doi: $10.1145 / 1219092.1219096$

[9] V. Danos et al. (2007): Distributed Measurement-based Quantum Computation. Electronic Notes in Theoretical Computer Science 170, doi:10.1016/j.entcs.2006.12.012.

[10] E. D'Hondt \& P. Panangaden (2005): Reasoning about quantum knowledge. In: Proceedings of FSTTCS '05, doi $10.1007 / 11590156 \_45$.

[11] E. D'Hondt \& M. Sadrzadeh (2011): Classical Knowledge for Quantum Security. Electronic Notes in Theoretical Computer Science 270(1), doi:10.1016/j.entcs.2011.01.014

[12] A. K. Ekert (1991): Quantum cryptography based on Bell's theorem. Physical Review Letters 67(6), doi $10.1103 /$ PhysRevLett.67.661.

[13] M. Elboukhari, M. Azizi \& A. Azizi (2010): Analysis of the Security of BB84 by Model Checking. IJNSA 2(2), doi $10.5121 /$ ijnsa.2010.2207

[14] R. Fagin et al. (2003): Reasoning About Knowledge. MIT Press.

[15] S. J. Gay, R. Nagarajan \& N. Papanikolaou (2008): QMC: A Model Checker for Quantum Systems. In: Proceedings of CAV 2008, doi:10.1007/978-3-540-70545-1_51.

[16] M. Kwiatkowska, G. Norman \& D. Parker (2004): Probabilistic symbolic model checking with PRISM: a hybrid approach. Int. J. Softw. Tools Technol. Transf. 6(2), doi:10.1007/s10009-004-0140-2.

[17] A. Lomuscio, H. Qu \& F. Raimondi (2009): MCMAS: a model checker for the verification of multi-agent systems. In: Proceedings of CAV '09, doi:10.1007/11691372_31.

[18] R. van der Meyden \& M. Patra (2003): Knowledge in Quantum Systems. In: Proc. of TARK '03, doi $10.1145 / 846241.846257$

[19] M. A. Nielsen \& I. L. Chuang (2000): Quantum Computation and Quantum Information. Cambridge University Press.

[20] M. Ying, Y. Li, N. Yu \& Y. Feng (2010): Model-Checking Linear-Time Properties of Quantum Systems. http://arxiv.org/abs/1101.0303. 